\title{
Percolation-dominated conductivity in a conjugated-polymer-carbon-nanotube composite
}

\author{
J. N. Coleman, S. Curran, A. B. Dalton, A. P. Davey, B. McCarthy, W. Blau, and R. C. Barklie \\ Department of Physics, Trinity College, Dublin University, Dublin 2, Ireland
}

(Received 19 May 1998)

\begin{abstract}
We have made electrical measurements on a system using carbon nanotubes as the dopant material. A semiconjugated, organic polymer was mixed with carbon nanotubes to form a wholly organic composite. Composite formation from low to high nanotube concentration increases the conductivity dramatically by ten orders of magnitude, indicative of percolative behavior. Effective mobilities were calculated from the spacecharge regions of the current-voltage characteristics for the 0-8\% mass fractions. After an initial rise these were seen to fall from 1-8\% doping levels as predicted by theory. From the values for conductivity and mobility, an effective carrier density was calculated. This was seen to decrease between $0 \%$ and $1 \%$, before rising steadily. [S0163-1829(98)51536-6]
\end{abstract}

The discovery two decades ago that polyacetylene could be made extremely conductive on doping with iodine or $\mathrm{AsF}_{5}$ (Ref. 1) was the initial step that indicated that conjugated polymers may be an important alternative to inorganic semiconductors as optoelectronic materials. However, conventional doping of polymers tends to quench their luminescent properties by introducing nonradiative trapping states such as solitons, polarons, and bipolarons. ${ }^{2}$ The key feature of conjugated polymers is the extended $\pi$-electron system in their backbone, which, due to Peierls distortion, results in a pseudoband gap. In these polymers conduction proceeds by variable range hopping of polarons. The band gap is approximately $1.5-2.5 \mathrm{eV}$, hence conductivity and mobility are generally low.

Carbon nanotubes, another form of conjugated carbon system, consist of one or more sheets of graphene wrapped around each other in concentric cylinders. Individually, nanotubes may be metallic or semiconducting, but in bulk they form a pseudometal with conductivity ${ }^{3}$ of approximately $10^{5} \mathrm{~S} / \mathrm{m}$. It is well known that the soot produced in a Krätschmer generator contains amorphous carbon, graphitic nanoparticles, and nanotubes. ${ }^{4}$ Ajayan et al. have shown that much of the superfluous carboniferous material can be burned away by heating in the presence of oxygen ${ }^{5}$ but yields are low. We have recently shown that a phenylenevinylene conjugated polymer can be used to extract the nanotubes from the other material in the fullerene soot. ${ }^{6}$ This process involves the formation of a polymer-nanotube composite. $^{7}$

Depending on the concentration of nanotubes, we can tailor the conductivity of this composite over many orders of magnitude. This can be achieved for any polymer by traditional doping with charge carriers, but dopants such as iodine make the material extremely reactive with oxygen so the polymer becomes very unstable. The nanotubes, however, interact with the polymer via weak van der $\mathrm{Waals}^{6}$ interactions so there is no decrease in stability in the composite. Introduction of nanotubes into the polymer also increases its strength, ${ }^{8}$ thermal conductivity, ${ }^{9}$ and optical stability. ${ }^{10}$ As a hard, conductive plastic this composite shows great potential as an antistatic coating or as electromagnetic shielding for electronic devices. It is the study of the exceptional electrical properties of this material to which this paper is devoted.

The polymer used in these studies $^{11}$ is $\operatorname{poly}(p$ phenlyenevinylene - co -2,5 - dioctoxy - $m$-phenylenevinylene) (PMPV), a variation on the more widely studied poly(paraphenylenevinylene), the repeat unit of which is depicted in Fig. 1. Synthesis of this polymer has been described previously. ${ }^{12}$ The nanotube powder was prepared in a Krätschmer generator, following a standard procedure as described extensively in the literature. ${ }^{13}$ Composite solutions were prepared for various mass fractions of nanotubes in PMPV. The nanotube powder and PMPV were mixed together in toluene and briefly sonicated. The mixture was then left to settle for approximately three days by which time some of the soot had precipitated. It must be pointed out that all mass fractions quoted are for the gross amount of soot added, and do not take into account the precipitated soot. Thin films for conductivity measurements were produced by spin casting with a Convac 1001 spin coater. The presence of a large amount of nanotubes in the film was confirmed by tunneling electron microscopy (TEM). The composite solution was spun onto a platinum electrode that had been vacuum deposited onto glass. The second platinum electrode was then sputtered onto the film to produce a $\mathrm{Pt}$ composite-Pt sandwich structure. The active area of each device was $4 \mathrm{~mm}^{2}$ and the film thickness of the order of a few hundred nanometers to a few micrometers. Contact to both electrodes was made using pressure probes and conductive paint. Dark conductivity measurements were made using a HP 4339-A voltage supply and current monitor. For all samples a two point probe method was used. Thickness measurements were made using a Zygo profilometer.

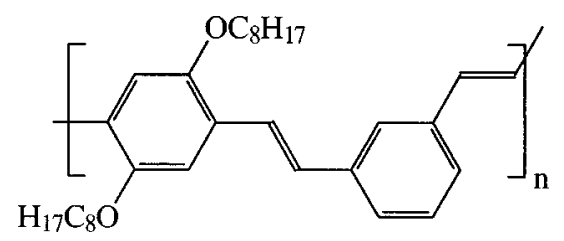

FIG. 1. The molecular structure of $\operatorname{poly}(p$-phenlyenevinyleneco-2,5-dioctoxy- $m$-phenylenevinylene). 




FIG. 2. A semilogarithmic plot of the conductivity of the composite for various mass fractions of nanotube powder in PMPV. The solid line is a fit from Eq. (1). Inset: Logarithmic current-voltage characteristic for the $8 \%$ sample, showing both Ohmic and quadratic regions.

As shown in Fig. 2, introduction of nanotubes to the polymer to form a composite can increase conductivity by up to ten orders of magnitude. We measured the conductivity of the pure PMPV to be $2 \times 10^{-10} \mathrm{~S} / \mathrm{m}$. However, it must be stressed that the conductivity of most polymers can vary substantially depending on molecular weight distribution, purity, conformation, and defect concentration. The composite conductivity was measured for different mass fractions of nanotube powder in PMPV for the range 0.5-36 \%. Up to 7\% the conductivity increases by approximately an order of magnitude from $2 \times 10^{-10} \mathrm{~S} / \mathrm{m}$ to $1.5 \times 10^{-9} \mathrm{~S} / \mathrm{m}$. Between $7 \%$ and $11 \%$ the composite displays a dramatic increase in conductivity by eight orders of magnitude to $1 \times 10^{-1} \mathrm{~S} / \mathrm{m}$. At higher concentrations this increase plateaus and conductivity of the $36 \%$ sample was measured to be $3 \mathrm{~S} / \mathrm{m}$. It is unlikely that the other carboniferous material in the nanotube powder plays a significant part in this large increase in conductivity as the conductivity of these particles is probably much less than that of the nanotubes.

This behavior is indicative of percolative character in composite systems. Percolation theory deals with the effect of varying, in a random system, the number of interconnections present. ${ }^{14}$ In this case the interconnections are the highly conductive nanotubes. Fourier $e t$ al. have proposed an analytical model, based on the Fermi-Dirac distribution, which describes the critical insulator to conductor transition: ${ }^{15}$

$$
\begin{aligned}
\log \left(\sigma_{c}\right)= & \log \left(\sigma_{n}\right) \\
& +\left[\log \left(\sigma_{p}\right)-\log \left(\sigma_{n}\right)\right] /\left[1+\exp \left\{b\left(p-p_{c}\right)\right\}\right],
\end{aligned}
$$

where $\sigma_{c}, \sigma_{n}$, and $\sigma_{p}$ are the composite, nanotube, and polymer conductivities, respectively, $p$ is the mass fraction, and $b$ is an empirical parameter that leads to the change in conductivity at the percolation threshold $p_{c}$. This equation is fitted to the data in Fig. 2. The best fit to the data gives $p_{c}$ $=8.4 \%$.

The inset in Fig. 2 shows a typical measured result for the current as a function of voltage. We can see two well-defined regions. The first is the low-field Ohmic region followed by a region quadratic in voltage. If the contact is Ohmic, the current is dominated, at high fields by carriers injected by the electrodes and known as space-charge limited current (SCLC). In order to ensure good Ohmic contact between electrodes and semiconductor, the work functions must be within $0.5 \mathrm{eV}$ of each other. As the work function of platinum is $5.65 \mathrm{eV}$ and that of the polymer is approximately 5.2 $\mathrm{eV}$ this should apply. ${ }^{16}$ This quadratic behavior only holds in the presence of shallow traps, or in the theoretical limit of no traps. A theoretical shallow trap SCLC is described by the Child-Langmuir equation: ${ }^{17}$

$$
J=9 \mu \varepsilon V^{2} / 8 l^{3},
$$

where $\mu$ is an effective mobility, $\varepsilon$ the permittivity, and $l$ the electrode spacing. This effective mobility is the product of the free carrier mobility and the fraction of free charge $\theta$. Hence we can calculate the effective mobility of carriers from the current-voltage characteristic. This effective mobility was measured for samples in the range $0-8 \%$. At higher concentrations the current flow is Ohmic at high fields, indicating pseudometallic behavior. The results are depicted in Fig. 3. The mobility of pure PMPV was $2 \times 10^{-10} \mathrm{~m}^{2} / \mathrm{V} \mathrm{s}$. On doping with $0.5 \%$ by mass nanotube powder this increases by a factor of a 100 , but subsequently falls steadily to a value of $8 \times 10^{-11} \mathrm{~m}^{2} / \mathrm{V}$ s for the $8 \%$ doped sample. The fall is due to the introduction of shallow trapping sites by the nanotubes, which tends to reduce $\mu_{\text {eff }}$ by decreasing the fraction of free charge $\theta$. Xu and Shen have shown theoretically that the effective mobility of carriers in a doped organic sys- 


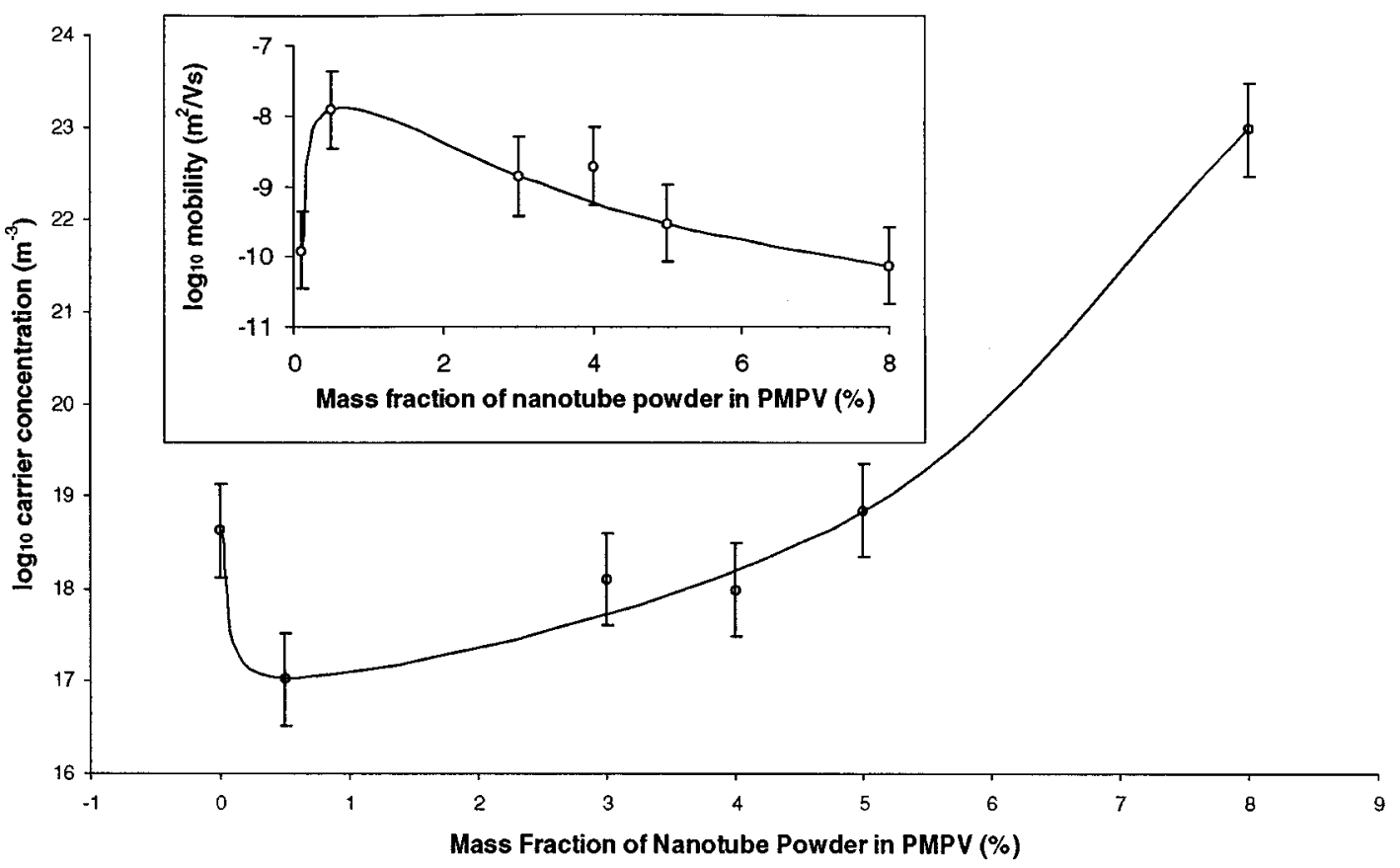

FIG. 3. A semilogarithmic plot of carrier concentration of the composite for various mass fractions of nanotube powder in PMPV. Inset: Semilogarithmic plot of effective mobility vs mass fraction of nanotube powder in PMPV.

tem will fall with increasing total trap density and hence doping level. ${ }^{18}$ This process is described by the equation

$$
\mu(H)=\mu_{0} a(1-b H),
$$

where $H$ is the total trap density and $a$ and $b$ are functions of temperature. An effective carrier density can be worked out from the effective mobility and conductivity. This is shown in Fig. 3. This carrier density was calculated for PMPV as $4 \times 10^{18} / \mathrm{m}^{3}$. However, for the $0.5 \%$ sample this falls to 1 $\times 10^{17} / \mathrm{m}^{3}$, before rising steadily to $1 \times 10^{23} / \mathrm{m}^{3}$ for the $8 \%$ sample. As polymers are predominately $p$ type $^{19}$ and graphitic structures can, under certain circumstances be $n$ type, ${ }^{20}$ this suggests that the nanotubes may introduce electrons to the polymer by a mechanism such as charge transfer, although further experiments are needed to clarify this. If this is the case, on doping, there will be a certain degree of recombination, resulting in carrier depletion for lower doping levels. At higher doping levels the electrons dominate and the composite becomes predominately $n$ type. Thus, at concentrations below the percolation threshold we see an increase in conductivity due to the introduction of carriers, even though there are no complete nanotube conductive paths through the system. This suggests that partial conductive paths must also have a role to play in the low concentration region. As we see a quadratic increase in current with voltage, even up to the $8 \%$ sample, some current is flowing through the polymer as we would not expect to see spacecharge effects in a pseudometal. Thus we suggest that the true percolation threshold, i.e., where the first complete nanotube conductive path occurs, lies between $8 \%$ and $9 \%$. It is probable that for the $8 \%$ sample, a mixed conduction process exists, with both carrier doping and partial conductive paths playing their parts. Hence, it is not clear whether the calculated carrier density for this concentration is meaningful.

It must be noted that we attempted to use the conjugated polymer PMMA to form a composite, but it was able to hold virtually none of the nanotube powder. We suggest that this has to do with the spatial conformation of the polymer and that nonlinear polymers such as PMPV can bond to the nanotubes while linear polymers such as PMMA cannot.

We conclude that doping of a semiconjugated polymer with carbon nanotubes to form an organic composite has been shown to increase the conductivity by ten orders of magnitude. This is mainly due to the introduction of conducting paths to the polymer. The behavior of conductivity as a function of doping is characteristic of percolation, with a threshold of approximately $8.5 \%$ mass fraction. The data fits well to an analytical model, describing conductivity in composites. After an initial increase, between $0 \%$ and $0.5 \%$, the effective mobility falls to below that of PMPV due to the introduction of traps by the nanotubes. The effective carrier density, as calculated from the conductivity and mobility, was seen to decrease between $0 \%$ and $2 \%$ before increasing steadily, indicating electron transfer from the nanotube to the polymer. As we observe space-charge limited currents up to the $8 \%$ doping level, it is reasonable to assume that the current increase below $8 \%$ is due to a combination of increased carrier donation by the nanotubes and the increase in partial conductive paths. The increase above is, however, due to the increase in number of complete conductive paths.

We would like to acknowledge many useful discussions with Michael Collins and Philippe Schaffnit. This work was supported by the European Union through TMR Contract No. Namitech, EBRFMRX-CT96-0067 (DG12-MIHT). 
${ }^{1}$ C. K. Chiang, C. R. Fincher, Y. W. Park, A. J. Heeger, H. Shirakawa, E. J. Lown, S. C. Gau, and A. G. MacDiarmid, Phys. Rev. Lett. 39, 1098 (1977).

${ }^{2}$ S. Roth, Synth. Met. 57, 3623 (1993).

${ }^{3}$ A. B. Kaiser, G. Düsberg, and S. Roth, Phys. Rev. B 57, 1418 (1998).

${ }^{4}$ D. Ugarte, MRS Bull. 1994, 39.

${ }^{5}$ P. M. Ajayan, T. W. Ebbesen, T. Ichihashi, S. Iijima, K. Tanigaki, and H. Hiura, Nature (London) 362, 522 (1993).

${ }^{6}$ A. P. Davey et al. (unpublished).

${ }^{7} \mathrm{~S}$. Curran et al. (unpublished).

${ }^{8}$ H. G. Wagner, O. Lourie, Y. Feldman, and R. Tenne, Appl. Phys. Lett. 72, 188 (1998).

${ }^{9}$ A. P. Ajayan et al. (unpublished).

${ }^{10}$ A. B. Dalton et al. (unpublished).

${ }^{11}$ W. Holzer, A. Penzkofer, S. H. Gong, A. Bleyer, and D. D. C. Bradley, Adv. Mater. 8, 974 (1996).
${ }^{12}$ S. Maier, Diploma Arbeit thesis, Trinity College, Dublin, July 1997.

${ }^{13}$ W. Kratschmer, L. D. Lamb, K. Fostiropoulos, and D. R. Huffman, Nature (London) 347, 354 (1990).

${ }^{14}$ R. Zallen, Physics of Amorphous Solids (Wiley, New York, 1983), Chap. 4.

${ }^{15}$ H. Baessler, N. Herrmann, N. Riehl, and G. Vaubel, J. Phys. Chem. Solids 30, 1579 (1969).

${ }^{16}$ J. Fourier, G. Boiteax, G. Seytre, and G. Marichy, Synth. Met. 84, 839 (1997).

${ }^{17}$ M. Pope and C. Swenburg, Electronic Processes in Organic Crystals (Clarendon, Oxford, 1982), p. 383.

${ }^{18}$ J. Xu and J. Shen, J. Appl. Phys. 83, 2646 (1998).

${ }^{19}$ J. L. Bredas and G. B. Street, Acc. Chem. Res. 18, 309 (1985).

${ }^{20}$ S. Curran, Electron. Prop. Fullerines, Int. Winterschool Electron. Prop. Novel Mater. 117, 427 (1993). 